\title{
From thermal to excited-state quantum phase transitions - the Dicke model
}

\author{
P. Pérez-Fernández ${ }^{1, *}$ and A. Relaño $o^{2, \dagger}$ \\ ${ }^{1}$ Departamento de Física Aplicada III, \\ Escuela Técnica Superior de Ingeniería, Universidad de Sevilla \\ ${ }^{2}$ Departamento de Física Aplicada I and GISC, Universidad Complutense de Madrid
} (Dated: today)

\begin{abstract}
We study the thermodynamics of the full version of the Dicke model, including all the possible values of the total angular momentum $j$, with both microcanonical and canonical ensembles. We focus on both the excited-state quantum phase transition, appearing in the microcanonical description of the maximum angular momentum sector, $j=N / 2$, and the thermal phase transition, which occurs when all the sectors are taken into account. We show that two different features characterize the full version of the Dicke model. If the system is in contact with a thermal bath and is described by means of the canonical ensemble, the parity symmetry becomes spontaneously broken at the critical temperature. In the microcanonical ensemble, and despite all the logarithmic singularities which characterize the excited-state quantum phase transition are ruled out when all the $j$-sectors are considered, there still exists a critical energy (or temperature) dividing the spectrum in two regions: one in which the parity symmetry can be broken, and another in which this symmetry is always well defined.
\end{abstract}

\footnotetext{
* pedropf@us.es

† armando.relano@gmail.com
} 


\section{INTRODUCTION}

Quantum phase transitions (QPTs) and critical phenomena play an important role in the study of many-body quantum systems [1]. During the last decade, a new kind of phase transition has been studied in depth - the excited-state quantum phase transition (ESQPT) [2 6]. In contrast to QPTs, which describe the non-analytical evolution of the ground state energy as a function of a control parameter, ESQPTs refer to a similar non-analytic behavior that takes place at a certain critical energy $E_{c}$, when the control parameter responsible for the QPT is kept fixed [7].

ESQPTs have been theoretically studied in many kinds of quantum systems. Paradigmatic examples are the Lipkin-Meshkov-Glick (LMG) [8, 9], the Dicke and Tavis Cummings models [10, 11], the interacting boson model [12], the molecular vibron model [13], atommolecule condensates [14], the kicked-top [15] or the Rabi model [16]. Also, a number of experimental results have been recently reported, in molecular systems [17], superconducting microwave billiards [18], and spinor condensates [19]. However, and despite the intense research performed during the last couple of years, some important questions still remain open. The most important one is whether the critical energy does separate two different phases in the spectrum. Contrary to what happens in quantum and thermal phase transitions, there are no clear traces of order parameters in ESQPTs. Though many physical observables become singular at the critical point, it seems impossible to find a magnitude which is zero at one side of the transition, and remains different from zero at the other (see, for example [5]). A recent proposal to characterize the transition relies on how the values of the physical observables change with energy [22]. This idea allows us to identify two different regions in the spectrum, but it does not provide an easy way to distinguish different phases just by measuring an appropriate observable. Another recent proposal relies on symmetry-breaking. A number of quantum systems showing ESQPTs are characterized by a discrete $Z_{2}$ symmetry which can be broken at one side of the transition, but not at the other. From fundamental physical reasons this seems a promising idea. First, it links ESQPTs with the breakdown of a certain symmetry, following a line of thought similar to the theory of thermal phase transitions. Second, this fact entails measurable dynamical consequences if a thermodynamic process is performed from a symmetry-breaking initial condition - the symmetry of the final equilibrium state remains broken only if the final 
energy is at the corresponding side of the transition, whereas the symmetry is restored on the contrary [20, 21]. However, if the initial condition has a well-defined value of this symmetry, nothing similar happens when crossing the critical energy. In other words, crossing an ESQPT does not entail a spontaneous breakdown of the corresponding symmetry under any circumstances; the ocurrence of this phenomenon depends on the details of the protocol.

Notwithstanding, the possible links between ESQPTs, thermal phase transitions, and the breakdown of certain fundamental symmetries of the system deserve to be explored. ESQPTs occur when the system is kept isolated from any environment, and thus can be described by means of the microcanonical ensemble. On the contrary, thermal phase transitions take place at a certain critical temperature $\beta_{c}$, and are usually described considering that the critical system is in contact with a thermal bath, that is, by means of the canonical ensemble [23]. But, as microcanonical and canonical descriptions become equivalent in the thermodynamical limit $N \rightarrow \infty$, where $N$ is the number of particles of the critical system, it is logical to expect that critical energy $E_{c}$ and critical temperature $\beta_{c}$ provide analogous information about the system. If we describe the critical system by means of the canonical ensemble, we should expect that the critical energy $E_{c}$ of the ESQPT corresponds to the internal energy $U=-\partial \log Z / \partial \beta$, evaluated at the critical temperature $\beta_{c}$, being $Z$ the canonical partition function. And if the system is described by means of the microcanonical ensemble, the critical temperature $\beta$ should correspond to the microcanonical temperature $\beta=\partial \log \rho(E) / \partial E$, evaluated at the critical energy $E_{c}$, being $\rho(E)$ the density of states. However, all the facts discussed below suggest just the opposite - that thermal and excitedstate quantum phase transitions are totally different. Probably, this is due to the fact that ESQPTs take place in systems with a small number of semiclassical degrees of freedom, implying that the size of the corresponding Hilbert space grows as $N^{f}$, being $f$ the number of degrees of freedom - the larger the number of degrees of freedom, the less important are the consequences of the ESQPT [5]. On the contrary, thermal phase transitions require an exponential growth of the size of the Hilbert space with the number of particles, in order to assure that intensive thermodynamical quantities, like the entropy per particle $S / N$ or the Helmholtz potential per particle $F / N$, are well defined in the thermodynamical limit. Hence, it is not clear even whether the correspondence between thermal and excited-state quantum phase transitions exists, or whether they are different phenomena occurring under different physical circumstances. Indeed, it is shown in Ref. [24] that, for collective systems, 
the thermodynamical limit $N \rightarrow \infty$ does not coincide with the true thermodynamic limit unless the number of degree of freedom $f$ also tends to infinity. So, ESQPTs and thermal phase transitions appear for different asymptotic regimes of $N$ and $f$. (We notice that, during the progress of this work, a similar analysis, but with a different aim, was performed in the generalized Dicke model, showing that it shows two different kinds of superradiance [25]).

In this work we tackle this task by studying, both analytically and numerically, the Dicke model. It describes a system of $N$ two-level atoms interacting with a single monochromatic electromagnetic radiation mode within a cavity [26]. It is well known from the seventies that this model exhibit a thermal phase transition [27, 28]. However, recently it was also found that undergoes an ESQPT [10] aside the QPT [29]. This kind of QPT has been experimentally observed in several systems [30], and the Dicke model itself can be simulated by means of a Bose-Einstein condensate in an optical cavity [31]. All these facts make this model the best one to study the relationship between thermal and excited-state quantum phase transitions.

Up to now, the majority of the works on the Dicke model, including the ones dealing with QPTs and ESQPTs (except [25], as we have pointed above), were done in the subspace with maximum pseudo-spin sector $j=N / 2$, in which the ground state is included. This restriction is enough to properly describe the recent experimental results [31], and also to study all the consequences of the QPT. Furthermore, ESQPTs have been observed in the subspace with $j=N / 2$, which can be described by means of a semiclassical approximation with just two degrees of freedom in the thermodynamic limit $N \rightarrow \infty$. However, it is well known that this restriction destroys the thermal phase transition [32]; the fact that the atomic subspace grows linearly with $N$ in the $j=N / 2$ sector makes impossible to properly define the entropy $S$ or the Helmholtz potential $F$, and therefore precludes the thermal phase transition. In this work we deal with the complete Dicke model, including all the $j$ sectors. This is equivalent to increase the number of degree of freedom in the system that eventually goes to infinity in the thermodynamical limit. Contrary to the seminal papers on the thermal phase transition [27, 28] we study the thermodynamics of this model in the microcanonical ensemble, considering the system isolated instead of being in contact with a thermal bath. This point of view allows us to study the possible connections between the excited-state and the thermal phase transitions. In particular, we show that each $j$ sector displays the same 
kind of ESQPT, provided that the coupling constant is large enough (see below for a detailed discussion regarding this condition), but having each one a different critical energy $E_{c}$. Paradoxically, this fact, together with the different weight of each $j$ sector in the spectrum of the complete Dicke model, destroys most of the signatures of the ESQPT, and somehow surprisingly entails the appearance of the typical signatures of thermal phase transitions, like the existence of an order parameter. In particular, we show that the collective contribution of all the $j$ sectors rules out the logarithmic singularities in the derivatives of the density of states, $\rho(E)$, and the third component of the angular momentum, $J_{z}$, characteristic of the ESQPT. However, one of the most important signatures of the ESQPT survives. The parity symmetry of the Dicke model (see below for details) can be still broken below the critical energy $E_{c}$, which exactly coincides with the canonical internal energy, $U$, evaluated at the critical temperature of the thermal phase transition, $\beta_{c}$. In the microcanonical ensemble, that is, if the system remains isolated from any environment, the expectation value of a symmetry-breaking observable, like $J_{x}$, is always zero above $E_{c}$, but it can be different from zero below; its particular behavior depends on the initial condition. If the system is in contact with a thermal bath, and is described by means of the canonical ensemble, a small symmetry-breaking term $\epsilon J_{x}$ produces that $\left\langle J_{x}\right\rangle \neq 0$, even if we take the limit $\epsilon \rightarrow 0$ after the thermodynamical limit is done. In other words, the symmetry-breaking observable $J_{x}$ plays here the same role than the magnetization in the Ising model; it is an order parameter of the transition, and shows that the parity symmetry becomes spontaneoulsly broken below the critical temperature. On the other hand, the behavior of an isolated system is different. As it happens if only the highly-symmetric sector, $j=N / 2$, is taken into account, the parity symmetry remains broken below the critical energy $E_{c}$ of the ESQPT only if this symmetry is yet broken in the initial condition. In other words, the behavior of the system is expected to be different depending on whether the system is heated by means of the Joule effect, or it is in contact with a thermal bath.

This paper is organized as follows. In section 2 we present the Dicke model. In section 3 we review the thermodynamics of the Dicke model restricted to the highly-symmetric Dicke states, $|j=N / 2, M\rangle$. We compare the results provided by the micro and canonical ensembles, and we analyze the symmetry-breaking character of the ESQPT. In section 4 we perform a similar analysis including all the $j$ sectors; we show that an ESQPT occurs in each $j$ sector. We also show that the symmetry-breaking nature of the transition is still present 
and leads to spontaneous symmetry-breaking if the system is in contact with a thermal bath. In addition, we study the main physical differences between the system in isolation and in contact with a thermal bath. Finally, we extract the more relevant conclusions in the last section.

\section{THE DICKE MODEL}

The Dicke model describes the interaction of $N$ two-level atoms of splitting $\omega_{0}$ with a single bosonic mode of frequency $\omega$, by means of a coupling parameter $\lambda$,

$$
H=\omega_{0} J_{z}+\omega a^{\dagger} a+\frac{2 \lambda}{\sqrt{N}} J_{x}\left(a^{\dagger}+a\right)
$$

where $a^{\dagger}$ and $a$ are the usual creation and annihilation operators of photons, and $\vec{J}=$ $\left(J_{x}, J_{y}, J_{z}\right)$ is the Schwinger pseudospin representation of the $N$ two-level atom system, that is, the total angular momentum of a system of $N 1 / 2$-spin particles. This Hamiltonian has two conserved quantities. The first one is $\Pi=\exp \left(i \pi\left[j+J_{z}+a^{\dagger} a\right]\right)$, due to the invariance of $H$ under $J_{x} \rightarrow-J_{x}$ and $a \rightarrow-a$; as this is a discrete symmetry, $\Pi$ has only two different eigenvalues, $\Pi\left|E_{i}, \pm\right\rangle= \pm\left|E_{i}, \pm\right\rangle$, and it is usually called parity. The second one is the total angular momentum $J^{2}$ of the $N 1 / 2$-spin particles. This entails that the Hamiltonian (10) is block-diagonal in $J^{2}$, and hence each sector is totally independent from the others. The main dynamical consequence is that each $j$ sector evolves independently in any protocol keeping the Dicke model isolated from any heat bath. Furthermore, as the recent experimental realizations of this model involve only the sector of maximum angular momentum, $j_{\max }=N / 2$, the great majority of the papers published during the last couple of years are devoted to this case.

This model shows QPTs, ESQPTs and thermal phase transitions. In the following paragraphs we summarize the known results.

\section{THE CASE WITH $j=N / 2$}

In this section, for the sake of completeness, we review the thermodynamics of the Dicke model restricted to the highly-symmetric Dicke states, $|j=N / 2, M\rangle$. This configuration corresponds to a two-level system in which $N$ bosons can occupy either the upper or the 
lower level [32]. It has been recently explored by means of a Bose Einstein condensate in an optical cavity [31]. First of all, we present the density of states $\rho(E)$, which is computed by means of the microcanonical ensemble, and later we show the same $\rho(E)$ but considering the calculation in the canonical ensemble. These are well established results. Finally, we compare both approaches and get some conclusions.

\section{A. Microcanonical ensemble}

Let's consider that the system is thermally isolated and that we perform the following procedure: first, we freeze the system keeping fixed all the external parameters of the Hamiltonian, up to it is equilibrated at $T \sim 0$. This entails that the ground state, which always correspond with the sector of maximum angular momentum $j=N / 2$, is the only populated energy level. Second, we perform a quench, abruptly changing one of the external parameters. Then, if the system remains thermally isolated from the environment, the unitary evolution is totally captured by the sector with $j=N / 2$. Hence, all the thermodynamic results after the system is equilibrated at the final values of the external parameters should be obtained from a microcanonical calculation with fixed $j=N / 2$. This calculation can be completed by means of a semiclassical approximation, following different methods [11, 33, 34]. Here, we follow the method in ref. [34].

Considering $\omega=\omega_{0}=1$, the density of states reads

$$
\rho(E, j)=\left\{\begin{array}{l}
2 j \text { if } E / N>1 / 2 \\
\left(\frac{E}{j}+1\right) j+\frac{2}{\pi} \int_{E / j}^{y_{+}} d y \operatorname{acos}\left(\frac{\sqrt{y-E / j}}{2 \lambda^{2}\left(1-y^{2}\right)}\right) \text { if }-1 / 2 \leq E / N \leq 1 / 2 \\
\frac{2}{\pi} \int_{y_{-}}^{y_{+}} d y \operatorname{acos}\left(\frac{\sqrt{y-E / j}}{2 \lambda^{2}\left(1-y^{2}\right)}\right) \text { if } E / N<-1 / 2
\end{array}\right.
$$

where

$$
y_{-}=-\frac{j+\sqrt{j} \sqrt{j+8 E \lambda^{2}+16 j \lambda^{4}}}{4 j \lambda^{2}}
$$

and

$$
y_{+}=\frac{-j+\sqrt{j} \sqrt{j+8 E \lambda^{2}+16 j \lambda^{4}}}{4 j \lambda^{2}}
$$


For the third component of the angular momentum, we obtain

$$
\frac{J_{z}(E, j)}{j}=\left\{\begin{array}{l}
0 \text { if } E / N>1 / 2 \\
\left(\frac{E^{2}}{2 j^{2}}-\frac{1}{2}\right) \frac{j}{\rho(E, j)}+\frac{2 j}{\pi \rho(E, j)} \int_{E / j}^{y_{+}} d y y \operatorname{acos}\left(\frac{\sqrt{y-E / j}}{2 \lambda^{2}\left(1-y^{2}\right)}\right) \text { if }-1 / 2 \leq E / N \leq 1 / 2, \\
\frac{2 j}{\pi \rho(E, j)} \int_{y_{-}}^{y_{+}} d y y \operatorname{acos}\left(\frac{\sqrt{y-E / j}}{2 \lambda^{2}\left(1-y^{2}\right)}\right) \text { if } E / N<-1 / 2
\end{array}\right.
$$

Finally, for the first component of the angular momentum and considering that the parity is totally broken in the initial state,

$$
\frac{J_{x}(E, j)}{j}=\left\{\begin{array}{l}
0 \text { if } E / N>-1 / 2, \\
\pm \frac{2 j}{\pi \rho(E, j)} \int_{y_{-}}^{y_{+}} d y\left(1-y^{2}\right) \text { acos }\left(\frac{\sqrt{y-E / j}}{2 \lambda^{2}\left(1-y^{2}\right)}\right) \text { if } E / N<-1 / 2,
\end{array}\right.
$$

where the sign depends on the initial state. This expression has been obtained taking into account only one of the two disjoint parts in which the semiclassical phase space is divided for $\lambda>\lambda_{c}$ and $E<-N / 2$ [20]. If the initial state has a well-defined parity, both parts of the semiclassical phase space are populated, giving rise to $\left\langle J_{x}\right\rangle=0$.

These results show that an ESQPT happens at $E_{c} / N=-1 / 2$ [10, 11, 20, 34]. There are singular points for both $\rho(E, j)$ and $J_{z}(E, j)$ - the derivatives of both magnitudes show a logarithmic divergence at $E_{c}$. The reason for this behavior is the following: the density of states, Eq. (2), is proportional to the size of the phase space available to the system,

$$
\rho(E, j)=C \int d q_{1} d q_{2} d p_{1} d p_{2} \delta\left[E-H\left(q_{1}, q_{2} ; p_{1}, p_{2}\right)\right]
$$

where $q_{1}$ and $q_{2}$ denote the semiclassical coordinates; $p_{1}$ and $p_{2}$, the semiclassical momenta, and $C$ is a normalization constant (see, for example, [34]). The key point is that despite this semiclassical system is finite, it describes the quantum Dicke model in the thermodynamical limit, $N \rightarrow \infty$, and it has just $f=2$ degrees of freedom. Furthermore, every quantum system showing an ESQPT is equivalent to a semiclassical system with a finite number of degrees of freedom (see, for example, ref. [5]). As a consequence, non-analyticities in the quantum density of states are linked to stationary points in the corresponding semiclassical model; and the geometric properties of such stationary points determine the nature of the corresponding singularities. In particular, systems with $f=1$ semiclassical degrees of freedom show logarithmic singularities in the density of states, as well as in certain physical 
observables at the critical energy of the ESQPT, $E_{c}$; systems with $f=2$ degrees of freedom show the same kind of singularities in the derivatives of the same magnitudes [5]. Results for an higher number of degrees of freedom have been recently published, showing that the larger $f$, the higher the derivative in which the logarithmic singularity takes place [35].

Also, if the parity symmetry is broken in the initial state, $J_{x}(E, j)$ acts like an order parameter for the ESQPT; that is, it shows a finite jump at $E_{c}$, from $\left\langle J_{x}\right\rangle \neq 0$ to $\left\langle J_{x}\right\rangle=0$ [20]. On the contrary, initial conditions with well-defined positive (or negative) parity do not suffer any change when crossing the ESQPT.

Another singular point is located at $E_{c} / N=1 / 2$, whilst its critical character is controversial [11, 34]. Above this energy, $\rho(E)=1$ and $\left\langle J_{z}\right\rangle=0$, due to the ergodic character of the atomic motion (now the whole phase space is accesible to the system). Despite this point is not usually identified as an ESQPT, it has some of the features of a second order phase transition. First, there exists an order parameter identifying two different phases: for $E<N / 2,\left\langle J_{z}\right\rangle \neq 0$, whereas $\left\langle J_{z}\right\rangle=0$ for $E>N / 2$. Second, there is a discontinuity in the derivative of $\rho(E)$, that is, in the second derivative of the cumulated level density $N(E)$. We will come back to this discussion in Sec. IV] Numerical results illustrating these facts are shown later.

\section{B. Canonical ensemble}

The same kind of calculation can be performed in the canonical ensemble, considering that the system weakly interacts with a thermal bath which commutes with $J^{2}$. Following ref. [32] we can obtain the partition function

$$
Z(N, \beta)=\frac{1}{\pi} \int_{-\infty}^{\infty} d y \exp \left(-\beta \omega y^{2}\right) \int_{-\infty}^{\infty} d x \exp \left(-\beta \omega x^{2}\right) Z_{g}(N, \beta),
$$

where

$$
Z_{g}(N, \beta)=\sum_{m=-N / 2}^{N / 2} \exp \left(-\beta m \sqrt{\omega_{0}^{2}+\frac{4 \lambda^{2} x^{2}}{N}}\right)
$$

The final result is

$$
Z(N, \beta)=\sqrt{\frac{1}{\pi \beta \omega}} \int_{-\infty}^{\infty} d x \exp \left[-\beta\left(\omega x^{2}+\frac{n}{2} \sqrt{\omega_{0}^{2}+\frac{4 \lambda^{2} x^{2}}{n}}\right)\right] \frac{\exp \left(\beta(n+1) \sqrt{\omega_{0}^{2}+\frac{4 \lambda^{2} x^{2}}{n}}\right)-1}{\exp \left(\beta \sqrt{\omega_{0}^{2}+\frac{4 \lambda^{2} x^{2}}{n}}\right)-1} .
$$


There is no way to write this integral in terms of simple analytical functions, but it can be evaluated numerically to obtain results for precise values of all the external parameters $\omega$, $\omega_{0}$ and $\lambda$. Furthermore, other thermodynamic results can be obtained from the partition function

$$
\begin{aligned}
\langle E\rangle & =-\frac{\partial \log Z}{\partial \beta}, \\
\left\langle J_{z}\right\rangle & =-\frac{1}{\beta} \frac{\partial \log Z}{\partial \omega_{0}} .
\end{aligned}
$$

In all the cases $\left\langle J_{x}\right\rangle=0$.

It has been shown that there is no thermal phase transition under these circumstances [32]. In other words, microcanonical and canonical ensembles give rise to totally different results. If the system remains thermally isolated there exists a critical energy $E_{c}=-N / 2$ at which a non-analiticity occurs, giving rise to a number of dynamical (and observable) consequences [10, 20]. On the other hand, if the system is put in contact with a thermal bath, everything changes smoothly with the temperature $\beta$; in particular, nothing happens at the critical temperature $\beta_{c}$, given by $\left\langle E\left(\beta_{c}\right)\right\rangle=-N / 2$.

\section{Results}

In this subsection, we compare the results of both the microcanonical and the canonical calculations, for a system with $\omega=\omega_{0}=1, \lambda=3 \lambda_{c}=1.5$, and $N=10^{5}$. All the results are plotted versus the scaled energy $\langle E\rangle / N$. For the canonical calculation, this energy is obtained directly from Eq. (11).

In Figs. 1, 2, and 3 we depict the results for $\left\langle J_{z}\right\rangle,\left\langle d J_{z} / d E\right\rangle$, and $\left\langle J_{x}\right\rangle / N$ respectively. In the first two cases, we show both the microcanonical (solid green points) and the canonical (dashed red line) calculations; in Fig. 3, we show just the microcanonical calculation, because $\left\langle J_{x}\right\rangle=0$ in the canonical ensemble. In all the cases we show the critical energy of the ESQPT, $E_{c} / N=-1 / 2$, by means of a vertical dashed line.

As a general result, we can observe that the behavior of the Dicke model in the $j=N / 2$ sector is totally different depending whether it is thermally isolated or in contact with a thermal bath. In the first case, we can see neat signatures of the ESQPT (a singular point in $\left\langle d J_{z} / d E\left(E_{c}\right)\right\rangle$, or the crossing from $\left\langle J_{x}(E)\right\rangle \neq 0$ to $\left\langle J_{x}(E)\right\rangle=0$, if the parity symmetry is broken in the initial state). In the second one, no traces of such phenomena are present. The 


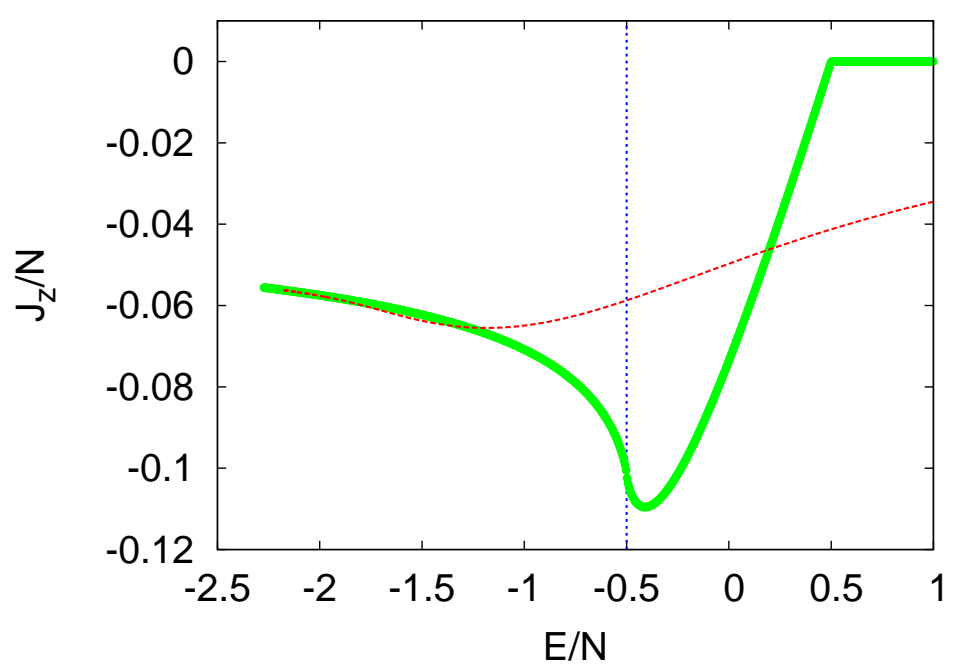

FIG. 1. (Color online) $J_{z}$ for the microcanonical ensemble (green solid points), and the canonical ensemble (dashed red line). The vertical dashed line shows the energy of the ESQPT.

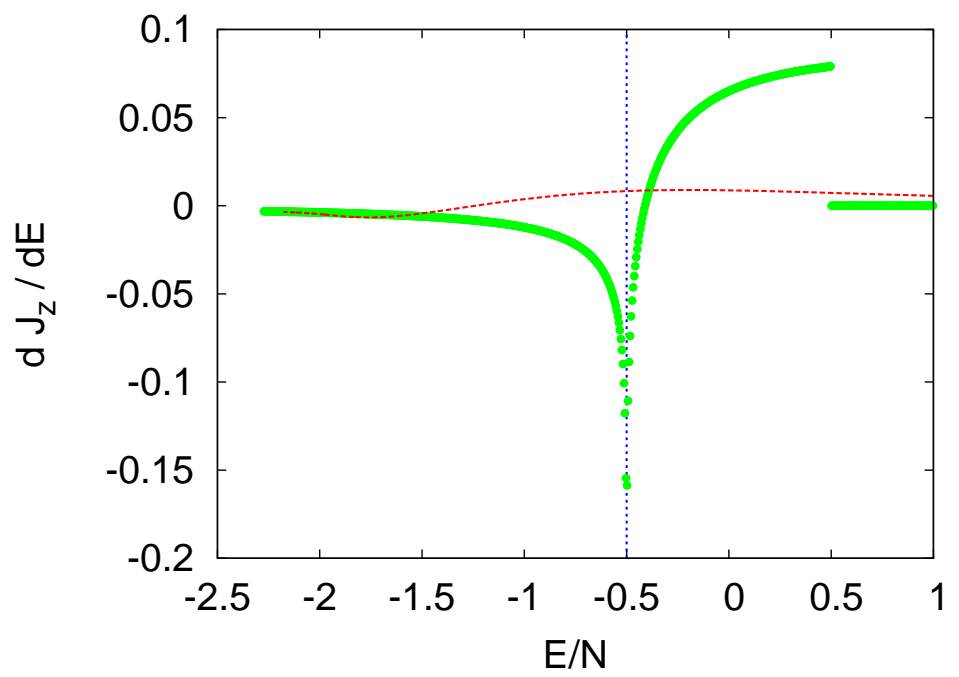

FIG. 2. (Color online) $d J_{z} / d E$ for the microcanonical ensemble (green solid points), and the canonical ensemble (dashed red line). The vertical dashed line shows the energy of the ESQPT.

reason behind this result is that the microcanonical density of states grows linearly with $N$. This entails that the thermodynamic magnitudes that should be extensive, like the entropy, $S$, or the Helmholtz free energy, $F$, grow with $\log N$, and therefore $S / N \rightarrow 0$ and $F / N \rightarrow 0$ in the thermodynamic limit. The main consequence is that the different ensembles are not equivalent in the thermodynamic limit, and that thermodynamics in this system is far from usual, and hence the results for the different statistical ensembles do not coincide. As it is 


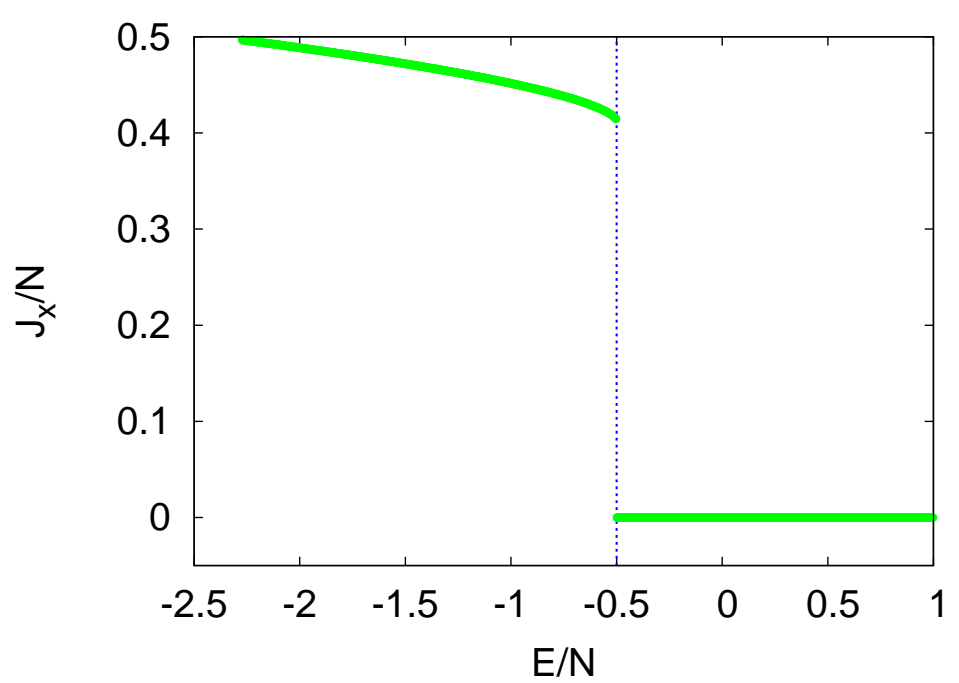

FIG. 3. (Color online) $J_{x}$ for the microcanonical ensemble (green solid points). The vertical dashed line shows the energy of the ESQPT.

pointed in [24] this is due to the finite number of (semiclassical) degrees of freedom that the system has in the thermodynamic limit, $N \rightarrow \infty$.

\section{THE FULL DICKE MODEL}

In this section, we perform a similar analysis as the former one, but now including all the $j$-sectors in the calculation. From a semiclassical point of view, this entails that the number of degrees of freedom $f$ also goes to infinity in the thermodynamical limit.

\section{A. Microcanonical ensemble}

If we consider that the system is thermally isolated, we can follow the same procedure than for the case with $j=N / 2$, taking into account that each $j$-sector is totally independent from the others. In other words, we can rely on the semiclassical approximation for each $j$ sector, and then collect all these results. Note, however, that the semiclassical approximation only gives good results for large values of the total number of two-level atoms, $N$. Hence, our procedure is questionable for sectors with low values of $j$, and, in particular, for the $j=0$ sector. This issue is discussed in detail later on.

To profit from the results obtained in the previous section, we proceed in the following 
way. The full Dicke model reads,

$$
H=\omega a^{\dagger} a+\omega_{0} J_{z}+\frac{2 \lambda}{\sqrt{N}} J_{x}\left(a+a^{\dagger}\right) .
$$

Considering that this Hamiltonian is block-diagonal in a $|j, M\rangle$ basis, the previous equation can be written as follows,

$$
H_{j}=\omega a^{\dagger} a+\omega_{0} J_{z}+\frac{2 \lambda}{\sqrt{2 j}} \sqrt{\frac{2 j}{N}} J_{x}\left(a+a^{\dagger}\right),
$$

where $H_{j}$ denotes the Hamiltonian $H$ in the sector with total angular moment equal to $j$. Thus, we can define an effective coupling constant for each $j$-sector, $\lambda_{j}^{\text {eff }}=\lambda \sqrt{2 j / N}$, giving rise to

$$
H_{j}=\omega a^{\dagger} a+\omega_{0} J_{z}+\frac{2 \lambda_{j}^{\mathrm{eff}}}{\sqrt{2 j}} J_{x}\left(a+a^{\dagger}\right) .
$$

From this result we conclude that the Hamiltonian of each $j$-sector, $H_{j}$, is formally identical than the one of the highly-symmetric sector, $j=N / 2$, but with a different effective coupling $\lambda_{j}^{\text {eff }}$.

With this in mind, we proceed to discuss the presence of ESQPTs in each $j$-sector. From the results derived in the section ПIA, we conclude:

1. ESQPT appears if $\lambda>\lambda_{c}=\sqrt{\omega \omega_{0}} / 2$. This entails that each $j$-sector requires a different coupling constant to show the ESQPT, the smaller the value of $j$, the larger the coupling,

$$
\lambda_{c}^{(j)}=\sqrt{\frac{N \omega \omega_{0}}{8 j}} .
$$

Therefore, the $j=0$ sector does not exhibit an ESQPT in any case $\left(\lambda_{c}^{(j)} \rightarrow \infty\right)$, and the lower values of $j$ require so large coupling constants for having ESQPTs, that these transitions are restricted to the larger values of $j$ in all the practical cases.

2. The critical energy for each sector is located at $E_{c}^{j} / N=-j / N$, and the energy of the other singular point at $E_{*}^{j} / N=j / N$. Thus, the lower $j$, the smaller is the energy band between these two singular points. If $j \rightarrow 0$ with a coupling constant large enough for the ESQPT to occur, the band shrinks to a single point located at $E / N=0$.

3. For any finite value of the coupling strenght in the superradiant phase, $\lambda>\lambda_{c}$, the dynamics of the full Hamiltonian is the result of collecting all the $j$-sectors, with both critical and non-critical behaviour. 
Considering that each $j$-sector is totally independent from the others, the density of states for the full Hamiltonian can be obtained as

$$
\rho(E)=\sum_{j=0}^{N / 2} g(N, j) \rho(E, j)
$$

being $g(N, j)$ the degeneracy of each $j$-sector, and $\rho(E, j)$ is given by Eq. (2).

The degeneracy is obtained as the number of ways in which a set of $N 1 / 2$-spin particles can give rise to a total angular momentum $j$. The result is

$$
g(N, j)=\frac{1+2 j}{1+j+N / 2}\left(\begin{array}{c}
N \\
N / 2-j
\end{array}\right) .
$$

To make easier the analytical calculations, it is preferable to work with an alternative version of this expresion. Instead of the angular momentum $j$, we consider the variable $x=j / N$, which can be taken as a continuous variable $x \in[0,1 / 2]$ in the thermodynamical limit, $N \rightarrow \infty$. Also, we write the combinatorial numbers in terms of the Gamma function, and therefore we obtain a continuous function $g(N, x)$ for any finite (but large) value of $N$,

$$
g(N, x)=\frac{(1+2 N x) \Gamma(N+1)}{\Gamma(1+N / 2-N x) \Gamma(2+N / 2+N x)} .
$$

Hence, the total density of states is given by

$$
\rho(E, N)=\int_{0}^{1 / 2} d x g(N, x) \rho(E, N x)
$$

We can apply the same procedure to the expected values of $J_{z}$ and $J_{x}$, obtaining

$$
J_{z}(E, N)=\frac{1}{\rho(E, N)} \int_{0}^{1 / 2} d x g(N, x) \rho(E, N x) J_{z}(E, N x),
$$

with $J_{z}(E, N x)$ given by Eq. (5). And

$$
J_{x}(E, N)=\frac{1}{\rho(E, N)} \int_{0}^{1 / 2} d x g(N, x) \rho(E, N x) J_{x}(E, N x),
$$

with $J_{x}(E, N x)$ given by Eq. ([6) . All these integrals have to be performed numerically since it is not possible to get analytical expressions.

As it has been pointed before, this procedure assumes that all the $j$ sectors can be properly described by means of the semiclassical approximation, and this is not completely true. Therefore, the goodness of the final result critically depends on the shape of Eq. (19). 
If the subsequent integrals are dominated by sectors with $j$ large enough, we can rely on our procedure; if they are dominated by the lowest $j$-sectors, the procedure is not going to work. So, prior to present the numerical results, we study here the shape of the function $g(N, x)$. Its maximum can be obtained by solving the equation $d g(N, x) / d x=0$. An asymptotic expansion when $N \rightarrow \infty$ and a Taylor expansion around $x=0$ show that this maximum is located at

$$
x_{\max }=\frac{1}{2 \sqrt{N}}-\frac{1}{2 N}+O\left(\frac{1}{N^{3 / 2}}\right) .
$$

This result imply two apparently contradictory consequences. First, the maximaly degenerated $j$-sector is

$$
j_{\max }=\frac{\sqrt{N}}{2}-\frac{1}{2}+O\left(\frac{1}{\sqrt{N}}\right) .
$$

Hence, $j_{\max } \rightarrow \infty$ in the thermodynamical limit, and consequently the semiclassical approximation used to derive Eqs. (21), (5) and (66) is expected to work provided that $N$ is large enough. On the contrary, it is also true that $x_{\max } \rightarrow 0$ when $N \rightarrow \infty$, suggesting that the maximally degenerated sector, and the one responsible of the behavior of the full Dicke Hamiltonian, is $j \rightarrow 0$, or the corresponding to the lower value of $j$ compatible with the given energy [25]. The solution of this apparent paradox is that $g(N, x)$ becomes non-continuous in the thermodynamical limit. Exact calculations from Eq. (19) show that

$$
\begin{aligned}
g(N, 0) & =\frac{2 \Gamma(1+N)}{(2+N) \Gamma(1+N / 2)^{2}}, \\
g\left(N, x_{\max }\right) & =\frac{N^{3 / 2} \Gamma(N)}{\Gamma\left(\frac{3-\sqrt{N}+N}{2}\right) \Gamma\left(\frac{3+\sqrt{N}+N}{2}\right)} .
\end{aligned}
$$

And the corresponding asymptotic expansion when $N \rightarrow \infty$ give rise to

$$
\begin{aligned}
g(N, 0) & \approx \frac{2^{3 / 2}}{\sqrt{\pi}} \frac{2^{N}}{N^{3 / 2}}, \\
g\left(N, x_{\max }\right) & \approx \frac{2^{3 / 2}}{\sqrt{\pi}} \frac{\mathrm{e}^{-1 / 2} 2^{N}}{N} .
\end{aligned}
$$

Therefore, the degeneracy of the $j_{\max }$-sector is larger than the degeneracy of the sector with $j=0$ for any finite size system with $N$ atoms, and the corresponding ratio is

$$
\frac{g(N, 0)}{g\left(N, x_{\max }\right)} \approx \frac{\mathrm{e}^{1 / 2}}{\sqrt{N}} \rightarrow 0, \quad \text { when } N \rightarrow \infty .
$$

In other words, $\lim _{N \rightarrow \infty} g\left(N, x_{\max }\right) \neq g(N, 0)$ despite $\lim _{N \rightarrow \infty} x_{\max }=0$, implying that $g(N, x)$ becomes non-continuous in the thermodynamical limit. Therefore, a rigurous calculation of the full density of states $\rho(E, N)$ and the corresponding expected values $J_{z}(E, N)$ 
and $J_{x}(E, N)$, requires to take this fact into account. Notwithstanding, from a practical point of view this is only important if we are interested in finite-size systems, or in obtaining finite-size corrections to the behavior in the thermodynamical limit. A first-order approximation for the behavior in the thermodynamical limit can be obtained just by considering the lower $j$-sector existing at a given energy $E$, which coincides with $j=0$ for $E / N>0$ [25]. In the next sections we will provide numerical results illustrating all these facts.

\section{B. Canonical ensemble}

Let's consider that the system is in contact with a thermal bath, so the total Hamiltonian (system + environment) reads

$$
H=H_{\text {Dicke }}+H_{\text {bath }}+H_{I},
$$

where $H_{I}$ is the interacting term between the system (the Dicke model) and its environment. If we assume that $\left[H_{I}, J^{2}\right] \neq 0$ and $\left[H_{I}, \Pi\right] \neq 0$, we have to take into account both parities and all the possible values of the angular momentum to derive the thermodynamics of the Dicke model. As it is indicated in [32], this is equivalent to a set of $N$ fermions occupying either the lower or the upper level of a two-level system. Under such circumstances, the partition function can be explicitely obtained; this calculation was completed around 40 years ago [28]. Here, we summarize the main results.

The partition function can be exactly derived, giving rise to

$$
Z(N, \beta)=\frac{2^{N}}{\sqrt{\pi \beta \omega}} \int_{-\infty}^{\infty} d x \exp \left(-\beta \omega x^{2}\right)\left[\cosh \left(\frac{\beta \sqrt{N \omega_{0}^{2}+16 \lambda^{2} x^{2}}}{2 \sqrt{N}}\right)\right]^{N} .
$$

This integral cannot be solved in terms of simple analytical functions. Exact results have to be derived by means of numerical integration. The same procedure can be used to obtain the expected values of the relevant observables of the system. For example, we can obtain $J_{x}$ and $J_{z}$ considering

$$
J_{\alpha}(N, \beta)=\frac{1}{Z(N, \beta)} \operatorname{Tr}\left[J_{\alpha} \exp (-\beta H)\right],
$$

where $\alpha=x, y, z$ is a label. From this equation it is straightforward to obtain

$J_{z}(N, \beta)=-\frac{\omega_{0} 2^{N-1}}{Z(N, \beta)} \sqrt{\frac{N^{3}}{\pi \beta \omega}} \int_{-\infty}^{\infty} d x \exp \left(-\beta \omega x^{2}\right) \frac{\left[\cosh \left(\frac{\beta \sqrt{N \omega_{0}^{2}+16 \lambda^{2} x^{2}}}{2 \sqrt{N}}\right)\right]^{N-1} \sinh \left(\frac{\beta \sqrt{N \omega_{0}^{2}+16 \lambda^{2} x^{2}}}{2 \sqrt{N}}\right)}{\sqrt{N \omega_{0}^{2}+16 \lambda^{2} x^{2}}}$ 
$J_{x}(N, \beta)=-\frac{N \lambda 2^{N-1}}{Z(N, \beta)} \sqrt{\frac{1}{\pi \beta \omega}} \int_{-\infty}^{\infty} d x x \exp \left(-\beta \omega x^{2}\right) \frac{\left[\cosh \left(\frac{\beta \sqrt{N \omega_{0}^{2}+16 \lambda^{2} x^{2}}}{2 \sqrt{N}}\right)\right]^{N-1} \sinh \left(\frac{\beta \sqrt{N \omega_{0}^{2}+16 \lambda^{2} x^{2}}}{2 \sqrt{N}}\right)}{\sqrt{N \omega_{0}^{2}+16 \lambda^{2} x^{2}}}$

Note that the last integral is an odd function in the $x$ variable, so $J_{x}(N, \beta)=0$. The same happens for any other symmetry-breaking observable, like, for example $q=\left(a+a^{\dagger}\right) / 2$. Also, both $\langle E\rangle$ and $\left\langle J_{z}\right\rangle$ can be obtained directly from the partition function making use of Eqs. (11) and (12).

Since phase transitions are defined in the thermodynamic limit, $N \rightarrow \infty$, we can apply Laplace's method to evaluate the partition function. Defining $y^{2}=x^{2} / N$ we can write

$$
Z(N, \beta)=\frac{\sqrt{N}}{\sqrt{\pi \beta \omega}} \int_{-\infty}^{\infty} d y \exp \left\{N\left[-\beta \omega y^{2}+\log \left(2 \cosh \left[\frac{\beta \omega_{0}}{2} \sqrt{1+\frac{16 \lambda^{2} y^{2}}{\omega_{0}^{2}}}\right]\right)\right]\right\}
$$

As a consequence,

$$
\lim _{N \rightarrow \infty} Z(N, \beta)=\sqrt{\frac{2}{\beta\left|\Psi^{\prime \prime}\left(y_{0}\right)\right|}} \exp \left[N \Psi\left(y_{0}\right)\right]
$$

where

$$
\Psi(y)=-\beta \omega y^{2}+\log \left(2 \cosh \left[\frac{\beta \omega_{0}}{2} \sqrt{1+\frac{16 \lambda^{2} y^{2}}{\omega_{0}^{2}}}\right]\right)
$$

and $y_{0}$ is the value of $y$ which maximizes $\Psi(y)$.

A phase transition normally happens when the possition of the maximum $y_{0}$ changes at a certain critical tempertaure $\beta_{c}$. The easiest way to obtain $y_{0}$ is solving $\Psi^{\prime}\left(y_{0}\right)=0$, and evaluating $\Psi\left(y_{0}\right)$ for all the solutions. For the Dicke model, the trivial solution $y_{0}=0$ exists for all the temperatures and the values of the system parameters. Under certain circumstances, there also exists another solution,

$$
\frac{4 \lambda^{2}}{\omega} \tanh \left(\frac{\beta \omega_{0}}{2} \sqrt{1+\frac{16 \lambda^{2} y_{0}^{2}}{\omega_{0}^{2}}}\right)=\omega_{0} \sqrt{1+\frac{16 \lambda^{2} y_{0}^{2}}{\omega_{0}^{2}}} .
$$

Defining $z=\sqrt{1+16 \lambda^{2} y_{0}^{2} / w_{0}^{2}}$, the former equation reads,

$$
\tanh \left(\frac{\beta \omega_{0} z}{2}\right)=\frac{\omega \omega_{0}}{4 \lambda^{2}} z .
$$

It is important to note that, by definition, $z>1$.

As $-1<\tanh (z)<1 \forall z$, the former equation only has solutions if

$$
\lambda>\lambda_{c}=\frac{\sqrt{\omega \omega_{0}}}{2} .
$$


Furthermore, the only way for Eq. (39) having a solution for $z>1$ is that $\tanh \left(\frac{\beta z}{2}\right)>\frac{\omega \omega_{0}}{4 \lambda^{2}} z$ at $z=1$; if this condition does not hold, the right side of the equation is larger than the left for any $z>1$. Therefore, if

$$
\beta<\frac{2}{\omega_{0}} \tanh ^{-1}\left(\frac{\omega \omega_{0}}{4 \lambda^{2}}\right),
$$

the only solution of the problem is the trivial one $y_{0}=0$. On the contrary, if $\beta$ exceeds this value, there exists a non-trivial solution $\widetilde{y}_{0} \neq 0$. Evaluating $\Psi(0)$ and $\Psi\left(\widetilde{y}_{0}\right)$ we can see that $\Psi\left(\widetilde{y}_{0}\right)>\Psi(0)$ in all the cases. Therefore, the position of the maximum $y_{0}$ changes at the critical temperature

$$
\beta_{c}=\frac{2}{\omega_{0}} \tanh ^{-1}\left(\frac{\omega \omega_{0}}{4 \lambda^{2}}\right),
$$

entailing that the partition function becomes non-analytic at the critical temperature $\beta_{c}$.

Summarizing, if $\lambda<\lambda_{c}$, there is no thermal phase transition. At $\lambda=\lambda_{c}$, the phase transition takes place at $\beta \rightarrow \infty$, that is, at $T \rightarrow 0$; it constitutes a QPT. If $\lambda>\lambda_{c}$, there exists a thermal phase transition at a critical temperature $T_{c}=1 / \beta_{c}$. The values for $\langle E\rangle$ and $\left\langle J_{z}\right\rangle$ in the thermodynamical limit can be easily obtained making use of Eqs. (11) and (12).

\section{Spontaneous symmetry-breaking at the critical temperature}

Phase transitions are usually linked to the breakdown of a global symmetry of the Hamiltonian. Above the critical temperature, the stable phase have the same symmetries than the Hamiltonian; below, one of these symmetries becomes spontaneously broken. The main signature of this fact usually lays in the behavior of the order parameter. For example, the paradigmatic Ising model without external magnetic field is symmetric under the permutation of all the spins, but the system becomes spontaneously magnetizated below the critical temperature. The usual order parameter of this transition reflects this fact. In any symmetric state, the total magnetization $m=M / N$ is zero; however, $m$ becomes different from zero in the ferromagnetic phase.

The seminal papers on the superradiant phase transition in the Dicke model do not consider this feature. As we have discussed above, the Dicke model has a discrete $Z_{2}$ symmetry, the parity $\exp \left(i \pi\left[j+J_{z}+a^{\dagger} a\right]\right)$. The usual order parameters for the superradiant transition are either $J_{z}$ or $a^{\dagger} a$. These observables provide a good physical insight of the character of the transition: in the superradiant phase both the bosonic field and the upper level of 
the atomic system are macroscopically populated, even when $\beta \rightarrow \infty$, given rise to expected values $\left\langle J_{z}\right\rangle$ and $\left\langle a^{\dagger} a\right\rangle$ different from zero [27-29]. However, neither $J_{z}$ and $a^{\dagger} a$ break the parity symmetry. Thus, it is interesting to seek alternative order parameters playing the same role than the magnetization in the Ising model. A good one is $J_{x}$, which has been recently used to study the ESQPT in the highly-symmetric sector [20]. As $\left\langle J_{x}\right\rangle=0$ in any eigenstate with well-defined parity, the strategy to study the behavior of this observable when crossing the phase transition consists in introducing a small symmetry breaking term in the Hamiltonian,

$$
H_{\epsilon}=\omega a^{\dagger} a+\omega_{0} J_{z}+\frac{2 \lambda}{\sqrt{N}} J_{x}\left(a^{\dagger}+a\right)+\epsilon J_{x},
$$

and taking $\epsilon \ll \omega, \omega_{0}, \lambda$.

The partition function of this system can be obtained following the same strategy than in the previous section. In the thermodynamical limit,

$$
\lim _{N \rightarrow \infty} Z_{\epsilon}(N, \beta)=\sqrt{\frac{2}{\beta\left|\Psi_{\epsilon}^{\prime \prime}\left(y_{0}\right)\right|}} \exp \left[N \Psi_{\epsilon}\left(y_{0}\right)\right]
$$

where

$$
\Psi_{\epsilon}(y)=-\beta \omega y^{2}+\log \left(2 \cosh \left[\frac{\beta \omega_{0}}{2} \sqrt{1+\left(\frac{\epsilon+4 \lambda y}{\omega_{0}}\right)^{2}}\right]\right)
$$

and $y_{0}$ is the value that maximizes $\Psi_{\epsilon}(y)$. From this result, we can obtain the expected values of $J_{z}$ and $J_{x}$ by means of

$$
\begin{aligned}
& \left\langle J_{z}\right\rangle_{\epsilon}=\frac{1}{\beta} \frac{\partial \log Z_{\epsilon}}{\partial \omega_{0}}, \\
& \left\langle J_{x}\right\rangle_{\epsilon}=\frac{1}{\beta} \frac{\partial \log Z_{\epsilon}}{\partial \epsilon} .
\end{aligned}
$$

And finally, we can study both parameters in the limit $\epsilon \rightarrow 0$. It is worth to remark that this procedure entails that the thermodynamic limit is taken before the $\epsilon \rightarrow 0$ limit. Spontaneous symmetry breaking in phase transitions occurs because these limits do not commute, leading to a finite value of the symmetry-breaking order parameter even in the limit $\epsilon \rightarrow 0$.

In Fig. 4 we show the results for $y_{0},\left\langle J_{z}\right\rangle$ and $\left\langle J_{x}\right\rangle$, both for the normal Dicke model, and for the case with the symmetry-breaking term, considering the limit $\epsilon \rightarrow 0$ (see caption for details). We can see that including the symmetry-breaking term does not change the results for the critical temperature, $\beta_{c}$, the value of $y_{0}$ and the expected value for $J_{z}$. However, $\left\langle J_{x}\right\rangle$ 


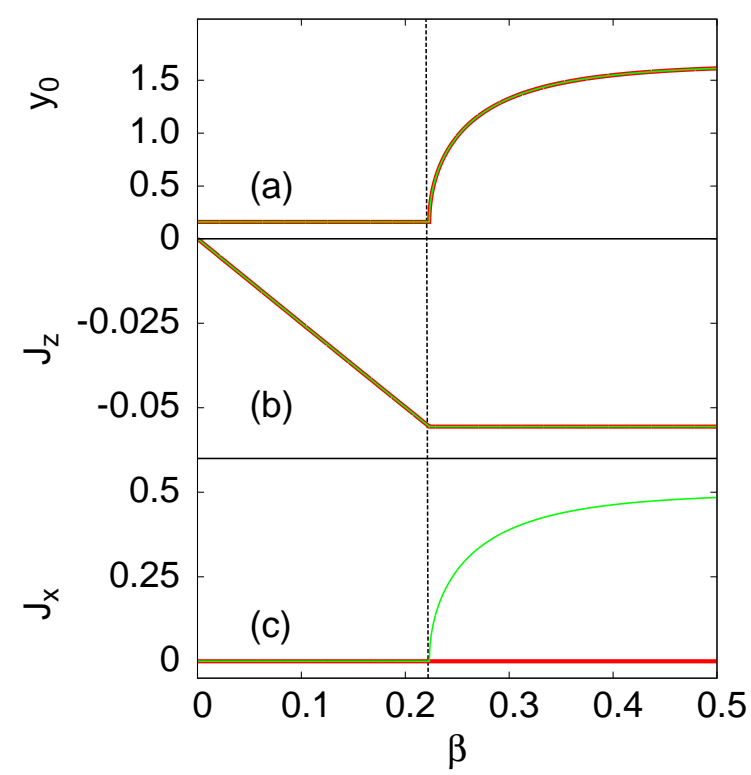

FIG. 4. (Color online) Value of $y_{0}$ and expected values of $J_{z}$ and $J_{x}$, both without the symmetrybreaking term (thick line, red online) and with the symmetry-breaking term, taking the limit $\epsilon \rightarrow 0$ (thin line, green online). Critical temperature $\beta_{c}$ is marked with a dotted vertical line.

changes dramatically: it is identically zero at both sides of the transition if the symmetrybreaking term is not included, but becomes different from zero in the superradiant phase if it is included, even if we take the $\epsilon \rightarrow 0$ limit. Hence, we conclude that the parity symmetry is spontaneously broken for $\beta>\beta_{c}$, and that $J_{x}$ is a good order parameter of the transition. Furthermore, this observable plays the same role than the magnetization in the paradigmatic Ising model.

Summarizing, from the results shown in this section we conclude that $J_{x}$ is the proper order parameter for the superradiant phase transition. In the following sections, we will compare this finding and the recently published results about symmetry-breaking and the ESQPT [20].

\section{Numerical results: different $j$-sectors}

Prior to study the ESQPT and the thermal phase transition, we give a glimpse about the behavior of the different $j$-sectors. In Fig. 5 we plot the results for the sectors $j=$ $2 N / 16,3 N / 16, \ldots, 8 N / 16$, with $\omega=\omega_{0}=1, \lambda=1.5$ and $N=10^{5}$. In particular, we deal with six different magnitudes: the density of states, $\rho(E)$; the derivative of the density of 

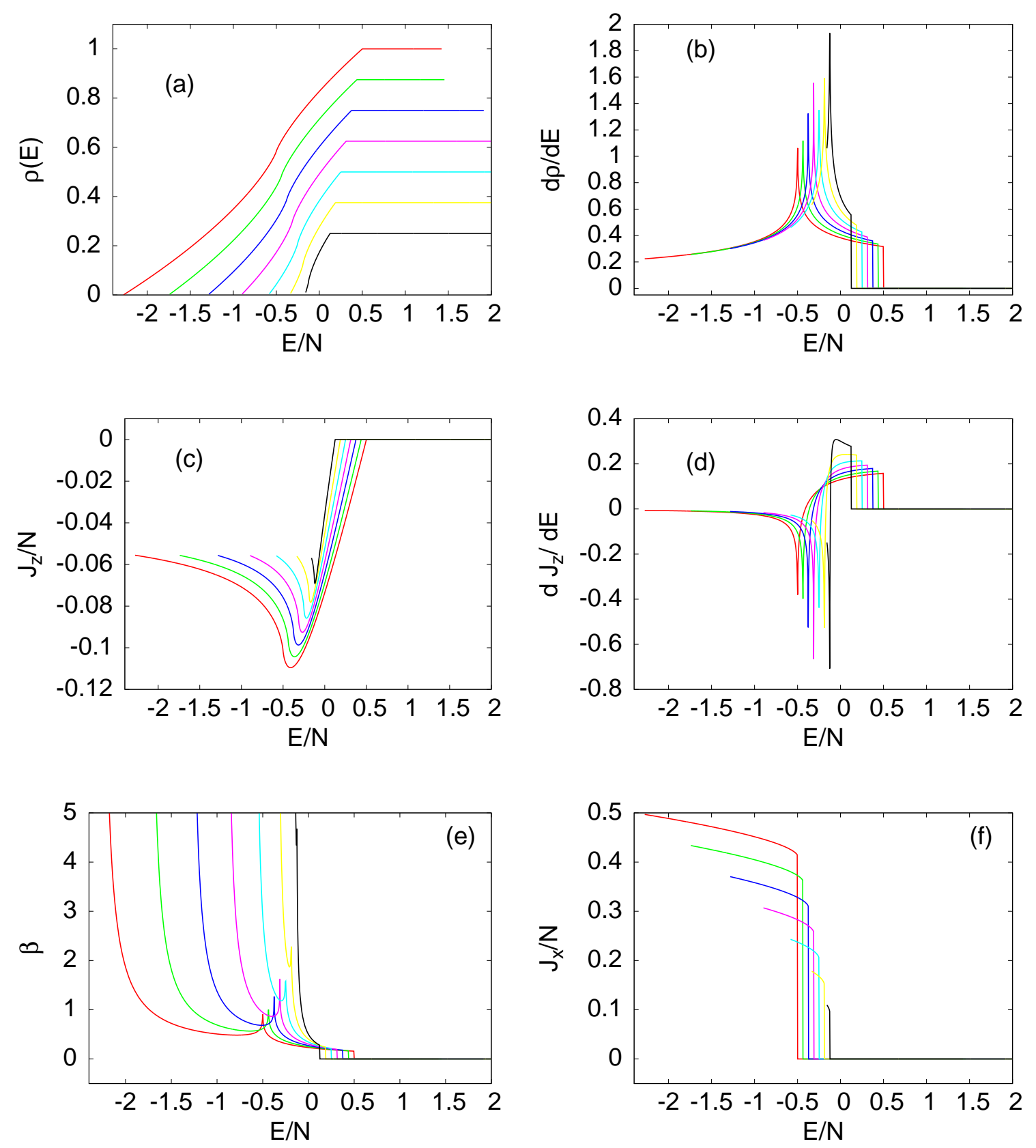

FIG. 5. (Color online) Microcanonical calculation for fixed and different values of $j$, for $N=10^{5}$. Panel (a), density of states, $\rho(E)$; panel (b), derivative of the density of states $\rho^{\prime}(E)$; panel (c), third component of the angular momentum, $\left\langle J_{z}\right\rangle$; panel $(\mathrm{d})$, derivative of the third component of the angular momentum $\left\langle d J_{z} / d E\right\rangle$; panel (e), temperature, $\beta$; panel (f), first component of the angular momentum, $\left\langle J_{x}\right\rangle$. Different colors show different values of $j, j=2 N / 16,3 N / 16, \ldots, 8 N / 16$.

states $\rho^{\prime}(E)$; the third component of the angular momentum, $\left\langle J_{z}\right\rangle$; the derivative of the third component of the angular momentum $\left\langle d J_{z} / d E\right\rangle$; the temperature, $\beta$; and the first 
component of the angular momentum, $\left\langle J_{x}\right\rangle$. All this magnitudes are calculated by means of the microcanonical formalism; $\beta$ is the microcanonical temperature

$$
\beta=\frac{\partial \log \rho(E)}{\partial E}
$$

We can see that the ESQPT occurs at a different energy for each different $j$-sectors. This is clearly seen in panels (b), (d), (e) and (f). The first three cases show logarithmic singularities associated with the derivatives of the density of states and the third component of the angular momentum [11]. It is worth to mention that this singularity is also present in the microcanonical temperature $\beta$. Also, note that $\beta$ is not a monotonous function of the energy; this is a clear signature of the anomalous thermodynamic behavior of each $j$-sector. Panel (f) shows the finite jump of the first component of the angular momentum, provided that the initial state has the parity symmetry broken [20].

All these facts give important hints to understand the behavior of the full Hamiltonian, including all the $j$-sectors. If the system remains thermally isolated and follows a non-trivial time evolution, for example resulting from a time-dependent protocol $\lambda(t)$, both the total angular momentum, $J^{2}$, and the parity, $\Pi$, are conserved. This entails that the evolution of every $j-\Pi$ sector is totally independent from the others. The main consequences of this fact are the following: $i$ ) every $j$-sector is affected by its ESQPT, showing the dynamical consequences reported in [10, 20]; ii) the behavior of the total system is the sum of all the sector, weighted by the corresponding degeneracies $g(N, x)$. In the next section we study the link between all these features and the thermal phase transition, well known since more than 40 years ago [28].

\section{E. Numerical results: ESQPT versus thermal phase transition}

In order to compare the physics of the isolated Dicke model (for which $J^{2}$ and $\Pi$ are conserved quantities) and the Dicke model in contact with a thermal bath (for which $J^{2}$ and $\Pi$ are not conserved), we proceed as follows. On the one hand, we obtain the microcanonical results, depending on the energy $E$, following the same procedure than in previous section. On the other, the canonical calculation depends on $\beta$, and the energy is derived from Eq. (11). It predicts a critical temperature, given by Eq. (39), and hence we can obtain the 
corresponding values for the critical energy,

$$
\left\langle E_{c}\right\rangle=-\left.\frac{\partial \log Z}{\partial \beta}\right|_{\beta_{c}}
$$

the critical value of $J_{z}$

$$
\left\langle J_{z, c}\right\rangle=\left.\frac{1}{\beta} \frac{\partial \log Z}{\partial \omega_{0}}\right|_{\beta_{c}}
$$

and the derivative of $J_{z}$

$$
\left\langle\frac{d J_{z, c}}{d E}\right\rangle=\left.\frac{d}{d E} \frac{1}{\beta} \frac{\partial \log Z}{\partial \omega_{0}}\right|_{\beta_{c}}=\left.\frac{\partial}{\partial \beta} \frac{1}{\beta} \frac{\partial \log Z}{\partial \omega_{0}} \frac{\partial \beta}{\partial E}\right|_{\beta_{c}} .
$$

With the values of the external parameters used in this work, $\omega=\omega_{0}=1$ and $\lambda=1.5$, we obtain

$$
\begin{aligned}
\beta_{c} & =0.223144, \\
\left\langle E_{c}\right\rangle / N & =-0.055, \\
\left\langle J_{z, c}\right\rangle & =-0.055=\left\langle E_{c}\right\rangle / N .
\end{aligned}
$$

The derivative of $J_{z}$ is not defined at the critical temperature $\beta_{c}$; it jumps from 0 to 1 .

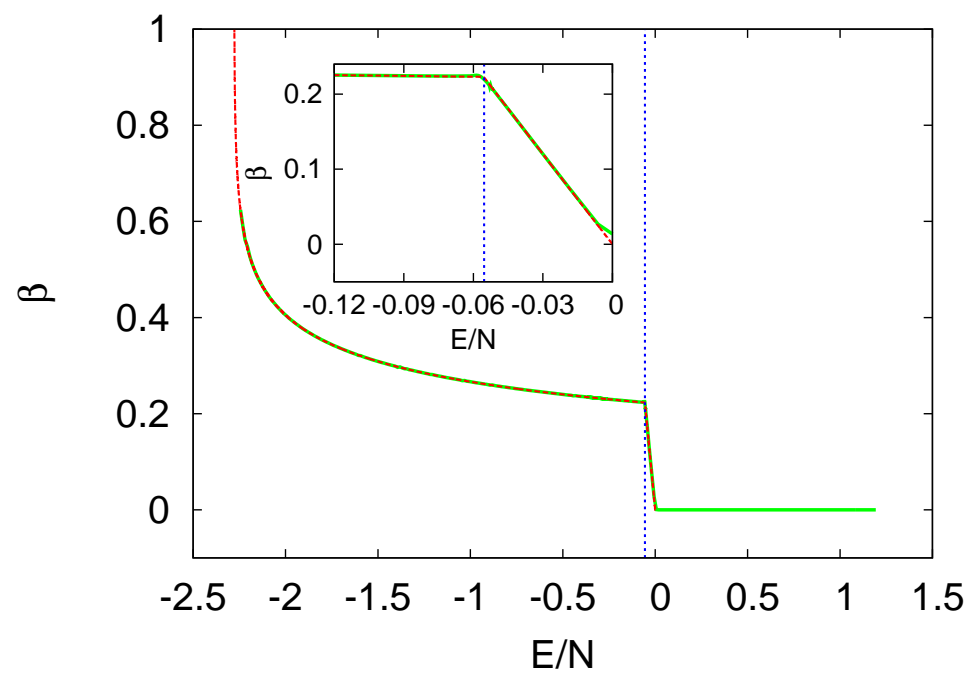

FIG. 6. (Color online) Temperature $\beta$ vs. energy $\langle E\rangle / N$ obtained by means of the microcanonical (solid green line) and the canonical (dahsed red line) ensemble. The vertical dashed line shows the critical energy $\left\langle E_{c}\right\rangle / N$. The inset shows the same results around this critical value.

In Fig. 6 we plot the temperature $\beta$ in terms of the energy $\langle E\rangle / N$. We display the microcanonical result by means of a solid (green online) line, and the canonical result by means 
of a dashed (red online) line. The critical value for the energy is shown by a vertical dashed (blue online) line, and the inset shows a zoom around the critical energy. Microcanonical calculation is done with $N=10^{5}$ particles. The canonical calculation is performed in the thermodynamical limit, by means of Laplace's method. The results are pretty different from the ones obtained with the different $j$ sectors. First, we can see that $\beta$ is a monotonous function of the energy, as one expects from standard thermodynamics. Second, microcanonical and canonical ensembles give rise to the same results; particularly, both display the same critical behavior. However, we can also see an important difference. When the system is put in contact with a thermal bath, the region with $\langle E\rangle / N>0$ is unreachable. In the canonical formalism, the limit $T \rightarrow \infty(\beta \rightarrow 0)$ corresponds with $\langle E\rangle / N \rightarrow 0$. Hence, if we heat the system by means an external source of heat, we are restricted to the region with $\langle E\rangle / N<0$. On the contrary, if the system remains isolated from any environment, and we heat the system by means of a mechanical procedure, for example performing fast cycles between $\lambda_{i}$ and $\lambda_{f}$, we can reach any final energy value. Note that $\langle E\rangle / N=0$ acts like a second critical energy, since the curve $\beta(E)$ shows a singularity at this point.

Another remarkable fact is that the logarithmic singularities shown in panel (e) of Fig. 5 are washed out - despite results shown in Fig. 6 consist of collecting all the $j$ sectors shown in panel (e) of Fig. 5, weighted by the corresponding degeneracy according to Eq. (19). On the other hand, the second singular point, taking place at $E_{*}^{j} / N=j / N$ in each $j$-sector, still occurs, at $E_{*} / N=0$.

Results for the third component of the angular momentum, $J_{z} / N$, are shown in Fig. 7. We can see the same kind on non-analiticity at the critical energy $E_{c} / N \sim-0.055$ than for the temperature $\beta$, despite the behavior for each $j$-sector, shown in panel (c) of Fig. 5, is totally different. Furthermore, both microcanonical and canonical calculations give the same results below $E_{*} / N=0$. At this value, the microcanonical ensemble shows a second singular point, and $J_{z} / N=0$ for $E / N>E_{*} / N$. It's worth to remark that, despite the consequences of the ESQPT are not so clear for this magnitude, the minimum appearing in each $j$-sector just above the critical energy $E_{c}^{j} / N$ is not visible in the figure, giving rise to an approximately flat region $J_{z} / N \sim-0.055$ for $E<E_{c}$. However, a zoom around $E_{c}$ shows that this minimum still exists for finite systems (see below for more details).

Results for the energy derivative of $J_{z}$ are shown in Fig. 8. Again, microcanonical and canonical ensembles give the same results, below $E_{*} / N=0$. In this case, we can see a finite 


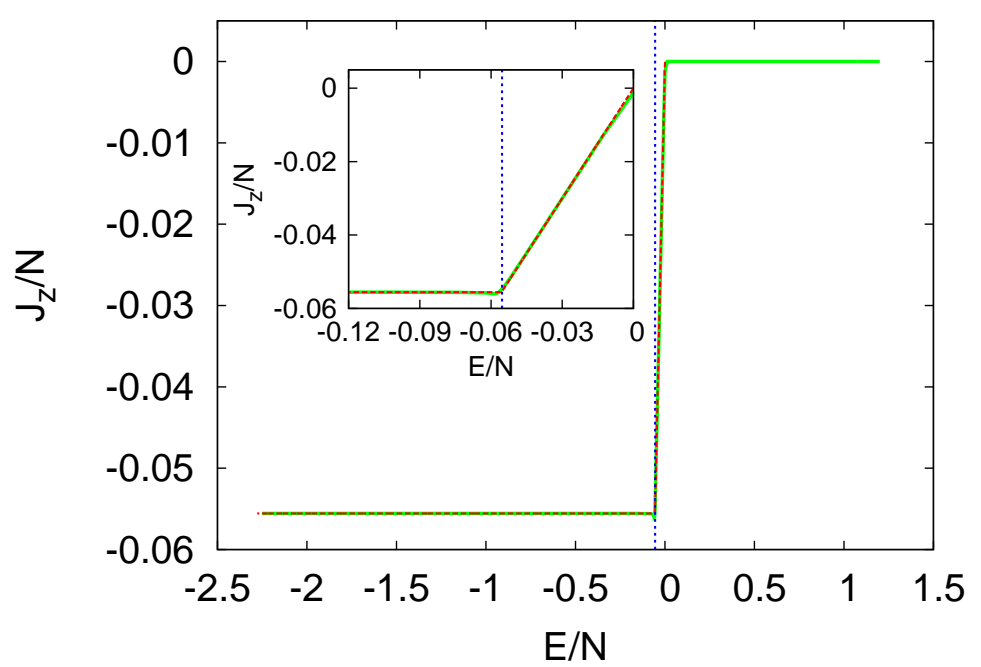

FIG. 7. (Color online) $J_{z}$ vs. energy $\langle E\rangle / N$ obtained by means of the microcanonical (solid green line) and the canonical (dahsed red line) ensemble. The vertical dashed line shows the critical energy $\left\langle E_{c}\right\rangle / N$. The inset shows the same results around this critical value.

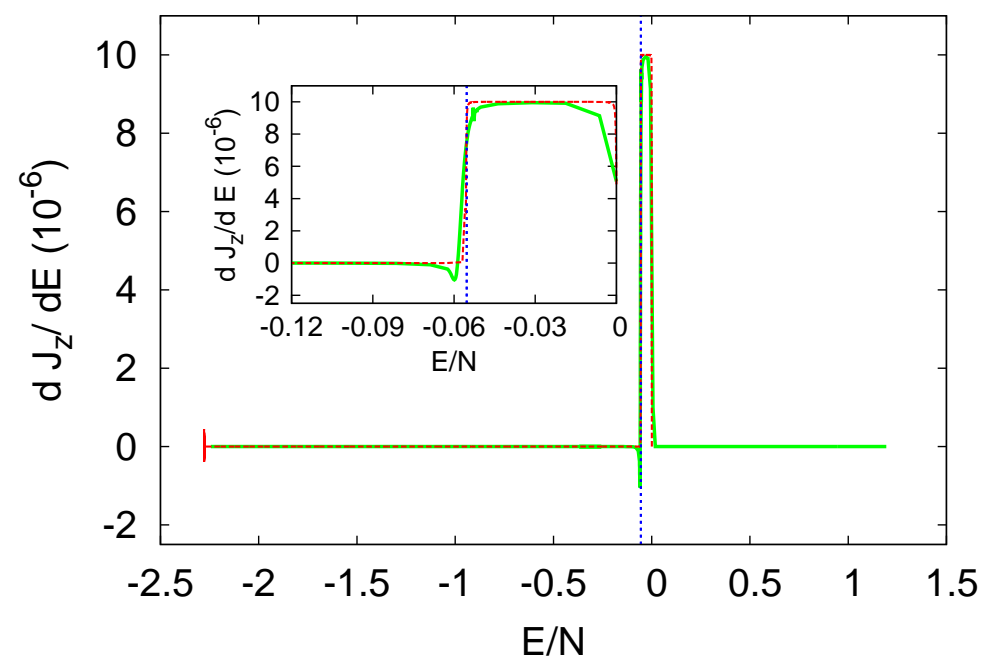

FIG. 8. (Color online) Derivative of $J_{z}$ vs. energy $\langle E\rangle / N$ obtained by means of the microcanonical (solid green line) and the canonical (dahsed red line) ensemble. The vertical dashed line shows the critical energy $\left\langle E_{c}\right\rangle / N$. The inset shows the same results around this critical value.

jump at the critical energy $E_{c}$; the logarithmic singularities, shown in panel (d) of Fig. 5 are also ruled out.

Finally, results for the first component of the angular momentum, $J_{x} / N$ are shown in Fig. 9. We depict the microcanonical result together with the calculation including the 


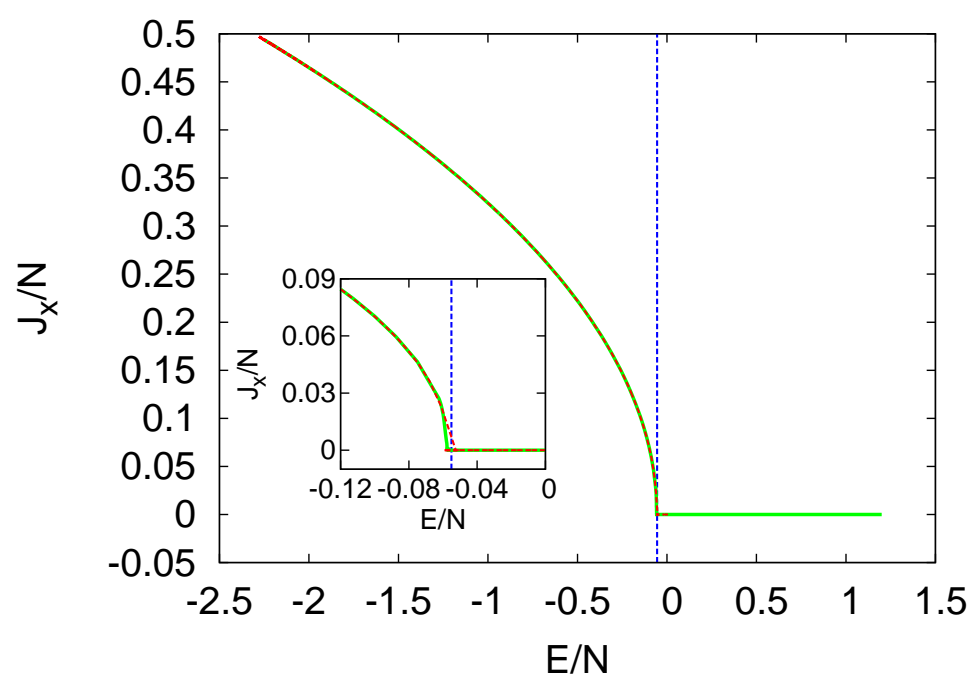

FIG. 9. (Color online) $J_{x}$ vs. energy $\langle E\rangle / N$ obtained by means of the microcanonical ensemble (solid green line) and the canonical ensemble with the symmetry-breaking term $\epsilon J_{x}$ (dashed red line). The vertical dashed line shows the critical energy $\left\langle E_{c}\right\rangle / N$. The inset shows the same results around this critical value.

symmetry-breaking term, $\epsilon J_{x}$, described in Sec. IVC. Microcanonical calculations have been done considering that the parity symmetry is totally broken in the initial state, and therefore the integrals over the phase space are restricted to one of the two disjoint regions existing when $E^{j} / N<E_{c}^{j} / N$ in each $j$-sector. (If we perform the calculations on the other disjoint region, we obtain the same curve, but with negative values for $\left.J_{x} / N\right)$. This observable shows a behavior that is qualitatively different than the previous ones. The main signature of the ESQPT is still present, but with a different qualitative behavior. $J_{x}$ is still an order parameter: it changes from $J_{x} \neq 0$ for $E<E_{c}$, to $J_{x}=0$ for $E>E_{c}$. The main feature of the full Dicke model is that this change in continuous, despite it is discontinuous in every $j$-sector experimenting the ESQPT.

From all these results, we infer the following conclusions:

1. Microcanonical and canonical ensembles are equivalent, below the singular point located at $E_{*} / N=0$. This energy constitutes an unreachable limit if the system is put in contact with a thermal bath. It corresponds to $\beta \rightarrow 0$ (or $T \rightarrow \infty)$. On the contrary, there is no such a limit if the system remains isolated.

2. The main signatures of the ESQPT are ruled out when we collect all the $j$-sectors: the 
logarithmic singularities in the derivatives of $\rho$ and $J_{z}$ are present when the system is neither isolated (microcanonical calculation) nor in contact with a thermal bath (canonical calculation). As these singularities are linked to stationary points in the corresponding semiclassical phase space, we can conclude that the relevance of such classical structures vanish when all the $j$-sectors are taken into account. A possible explanation, compatible with Ref. [24], is that, in this case, the number of effective degrees of freedom become infinite, since we have an infinite number of $j$-sectors (each one with $f=2$ degrees of freedom) in the thermodynamical limit.

3. Contrary to what happens with the other main signatures of the ESQPT, the breakdown of the $Z_{2}$ parity symmetry below the critical energy (or temperature), survives. If the system remains isolated from any environment, the system behaves as follows. Below the critical energy, $E<E_{c}$, the parity symmetry remains broken if it is broken in the initial condition; on the contrary, time evolution above the critical energy $E>E_{c}$ restores the symmetry [20, 21]. This entails that the expected value $\left\langle J_{x}\right\rangle$ keeps relevant information about the initial state. On the other hand, parity symmetry becomes spontaneously broken if the system is in contact with a thermal bath, as it is discussed in Sec. IVC, The most significative result shown in Fig. 9 is that this breakdown exactly coincides with the microcanonical result, when the integration over the phase space is restricted to one of the two disjoint regions existing for $E<E_{c}$. That is, thermal fluctuations make the system spontaneously choose one of these to possibilities. Hence, it is very worth to note the similarity in the behaviour of $\left\langle J_{x}\right\rangle$ in both the excited-state and the thermal quantum phase transitions, though the behavior of the system is not the same in isolation than in contact with a thermal bath.

\section{F. Results: finite size scaling}

Numerical results in the previous section have been obtained following different strategies. When the system is in contact with a thermal bath, that is, when we work in the canonical ensemble, we make the calculations in the thermodynamical limit, relying on the Laplace's method to evaluate the partition function. On the contrary, this limit is not explicitely done when the system is in isolation and microcanonical ensemble is considered. 
Furthermore, our method is applicable to finite-size systems, at least if they are large enough to apply the semiclassical approximation to each $j$-sector, and to consider that $x=j / N$ is very approximately a continuous variable $x \in[0,1 / 2]$. The aim of this section is to test the applicability of our results to systems small enough to be exactly solved by numerical diagonalization, and to profit from the analytical results to study the finite-size scaling of the critical behavior.

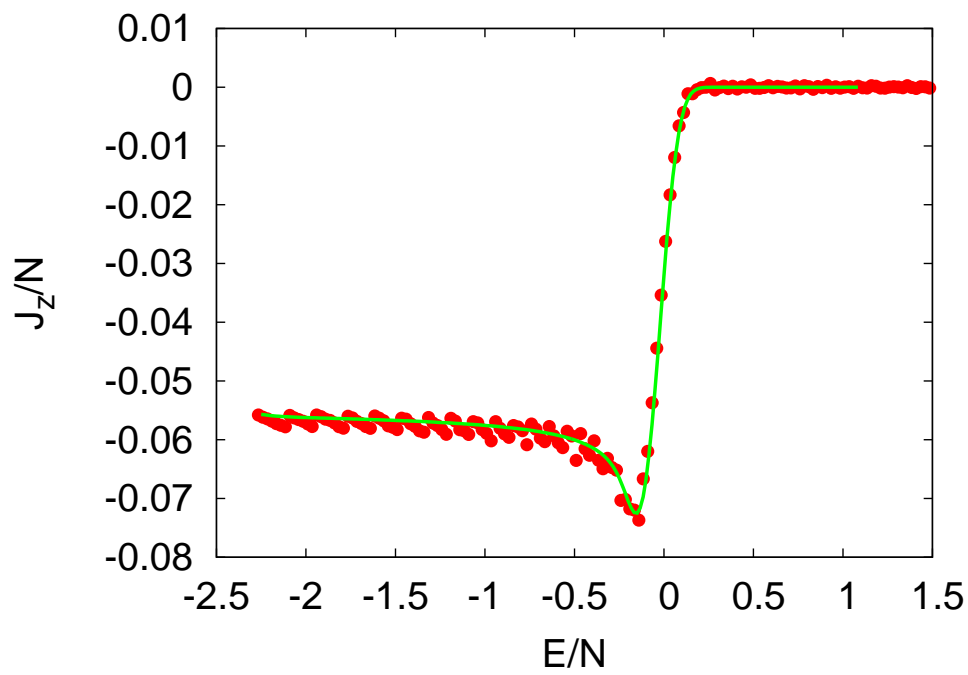

FIG. 10. (Color online) Exact numerical results (filled circles, red online) and microcanonical calculation (solid curve, green online) for the expected value of $J_{z} / N$, in a system with $N=50$ atoms.

In Fig. 10 we plot the numerical results for $\left\langle J_{z}\right\rangle$ obtained with a system with $N=50$ atoms including all the $j$-sectors, together with the microcanonical prediction given by Eq. (21). Numerical results have been obtained as follows. The Hamiltonian of each $j$ sector, $H_{j}$, is independently diagonalized. Then, the expected value in each eigenstate, $J_{z}(n, j)=\left\langle E_{n}^{j}\left|J_{z}\right| E_{n}^{j}\right\rangle$ is calculated. Finally, results for all the $j$-sectors are collected in an histogram with bins of size $\Delta E / N=0.05$, after considering the degeneracy of each sector, $g(N, j)$. As the actual number of photons is unbounded, the dimension of the Hilbert space is infinite, and hence the diagonalization procedure requires a truncation in the photonic Hilbert space. For all the calculations shown in this section, we have taken $n_{\max }=500$ photons, a number large enough to assure convergence in our results.

The match between theory and numerics is remarkable, taking into account all the approximations required to obtain the microcanonical result. At low energies, we see a kind of 


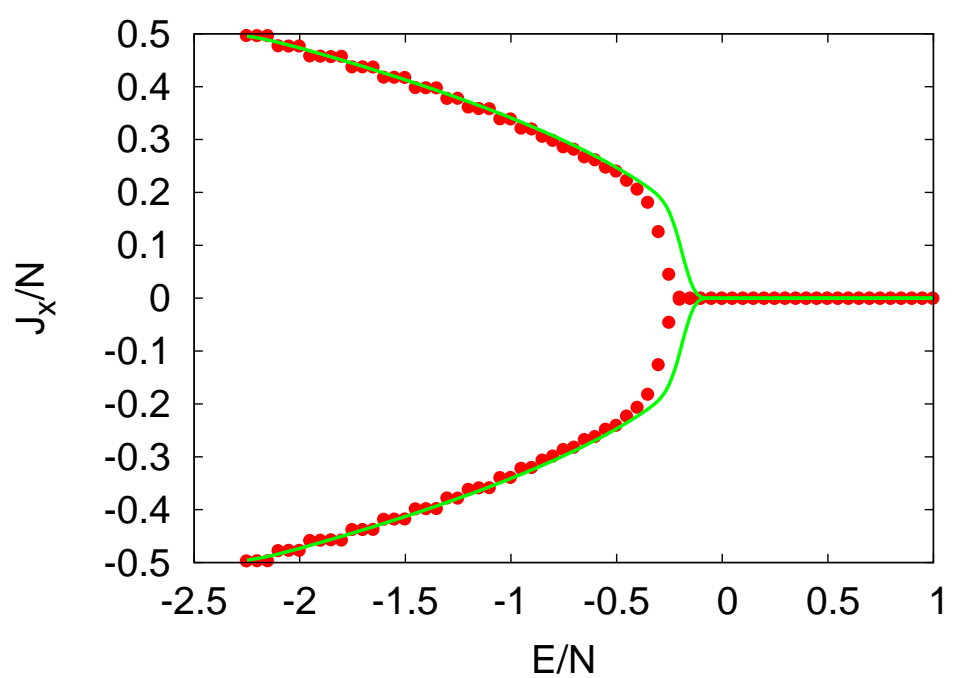

FIG. 11. (Color online) Exact numerical results (filled circles, red online) and microcanonical calculation (solid curves, green online) for the expected value of $J_{x} / N$, in a system with $N=50$ atoms. Numerical results have been obtained after introducing a small symmetry-breaking term, $\epsilon J_{x}$, with $\epsilon=10^{-6}$. For the analytical result, the symmetry-breaking term is not introduced, and the two disjoint regions of the phase space below the critical energy are integrated separately, to obtain the two branches of the theoretical curve.

saw-tooth structure in the numerical results, which is a consequence of the integrable nature of the low-lying spectrum of the Dicke model [36]. Besides this fact, the microcanonical results give a perfect description of the model. It is worth to remark the presence of a small dip close to the critical energy of the ESQPT. As it is discussed below, this dip is a remanent of the ESQPT and vanishes in the thermodynamical limit.

In Fig. 11we plot the results for $J_{x}$, obtained by means a procedure similar to the previous one. In this case, a small symmetry-breaking term, $\epsilon J_{x}$, with $\epsilon=10^{-6}$, has been introduced for the numerical diagonalization. As a consequence, the (almost) exact degeneracy of energy levels below $E_{c}$ is broken; in this phase, the spectrum consists of doublets, one level with $\left\langle J_{x}\right\rangle>0$, and another with $\left\langle J_{x}\right\rangle<0$, both with the parity symmetry totally broken, $\langle\Pi\rangle=0$. Hence, to collect the results for all the $j$-sectors, we have done two different histograms, one including all the levels with $\left\langle J_{x}\right\rangle>0$ and the other including the levels with $\left\langle J_{x}\right\rangle<0$. Also, two microcanonical integrals, Eq. (22), are performed, each one restricted to the corresponding disjoint region of the energy surface. It is worth to remark that the microcanonical integrals have been performed whithout including the symmetry-breaking 
term. Above the critical energy, only one integration region is considered, since the energy surface is not splitted anymore. In this region, the numerical calculations show that every eigenstate has well defined parity, despite the small symmetry-breaking term introduced in the Hamiltonian, and that the expected value of $J_{x}$ is always zero. All these facts are visible in Fig. 11. The match between numerical and microcanonical results is very good, except in the very surroundings of the critical energy. As large finite-size effects for this observable have been observed in the highly-symmetric sector [20], the small discrepancies observed in the figure are not surprising.

We can profit from the previous results to perform a finite-size scaling analysis of the transition. In particular, we rely on the theoretical expresions for the microcanonical ensemble to study how the statistical results depend on the system size $N$. Results for the finite-size precursor of the critical energy $E_{c}^{(N)}$ are shown in Fig. 12, We plot the difference between this precursor and the critical energy obtained by means the canonical calculation, $E_{c}^{(N)}-E_{c}$ versus the size of the system, in a double logarithmic scale. We also show a straight line representing the power-law behavior $E_{c}^{(N)}-E_{c} \propto N^{-\alpha}$, with $\alpha\left(J_{z}\right) \sim 0.47$, and $\alpha\left(J_{x}\right) \sim 0.41$. Calculations have been performed as follows. In the left panel, $E_{c}^{(N)}$ is estimated as the energy corresponding to the minimum of $J_{z} / N$. Though not explicitely shown, this minimum becomes less pronounced as the system-size grows, vanishing in the thermodynamical limit. In the right panel, $E_{c}^{(N)}$ is identified as the energy at which $J_{x} / N$ becomes less than 0.01. This bound is arbitrary, but we are not interested in quantitative results for each system size $N$, but in their scaling with the system size. From the results shown in Fig. 12, we can conclude that the finite size precursor $E_{c}^{(N)}$ tends to the critical energy $E_{c}$, with a power-law finite-size scaling.

In Fig. 13 we show the same results for the critical value of the third component of the angular momentum, $J_{z, c}^{(N)}-J_{z, c}$. Though in this case the scaling is not so clean, we still can conclude that $J_{z, c}^{(N)}-J_{z, c} \propto N^{-\alpha}$, with $\alpha \sim 0.40$.

\section{CONCLUSIONS}

In this work we have analyzed the relationship between the thermal phase transition and the ESQPT in the Dicke model. First of all, we have studied the thermodynamics of the model by means of microcanonical and canonical ensembles, and we have found that 

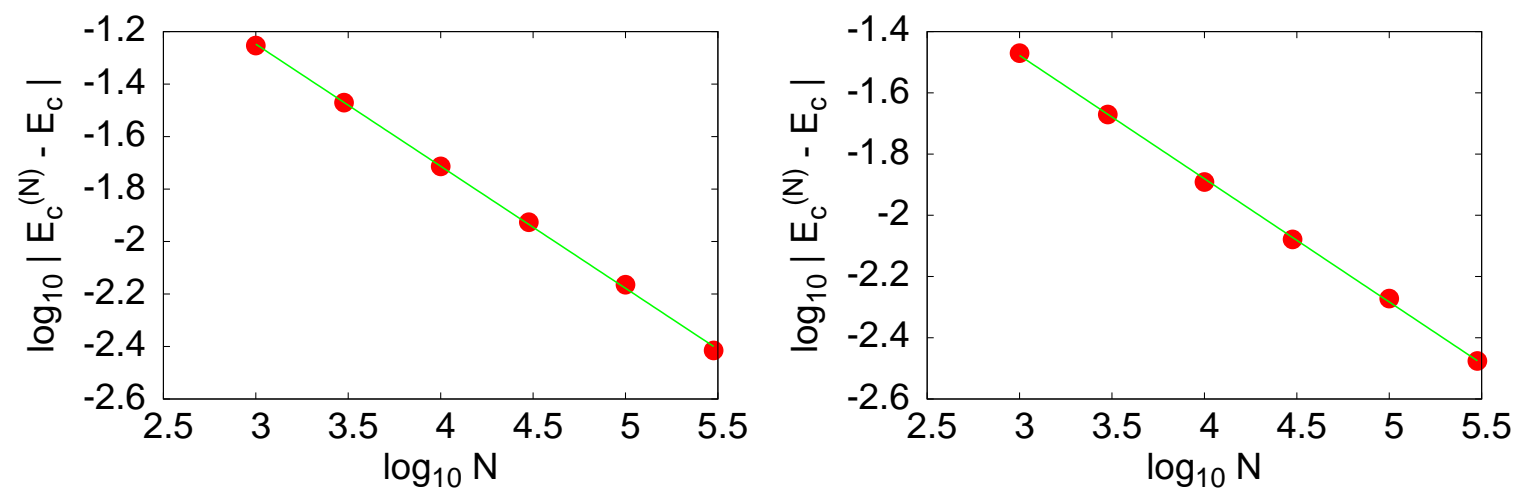

FIG. 12. (Color online) Finite size scaling for the critical energy, obtained with $J_{z}$ (left panel) and $J_{x}$. Both cases are depicted in a double logarithmic scale. The solid lines represent the least-square fits to straight lines, showing a power-law scaling with the system size.

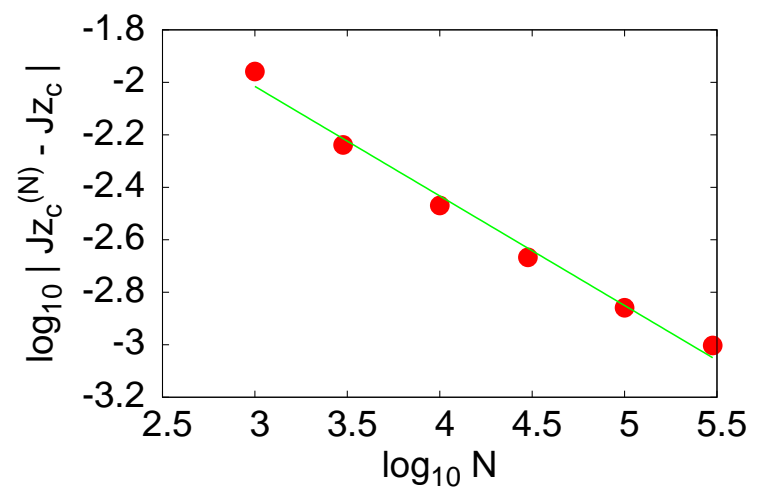

FIG. 13. (color online) Finite size scaling for the critical energy value of $J_{z}$, in a double logarithmic scale. The solid lines represent the least-square fits to straight lines, showing a power-law scaling with the system size.

both approaches are incompatible if we considerer just the highly-symmetric representation, i. e. $j=N / 2$. The reason is that the size of the Hilbert space grows linearly with the number of atoms, $N$, instead of exponentially. The main consequence is that extensive thermodynamic magnitudes, like the entropy $S$ or the Helmholtz potential $F$, do not scale with the number of particles $N$; thermodynamics is anomalous and the different ensembles are not equivalent in the thermodynamic limit, $N \rightarrow \infty$. In order to get a correct description of the thermodynamics properties it is necessary to include all the $j$-sectors.

To perform the microcanonical calculation including all the $j$ sectors, we have considered that all them can be adequately described by means of the semiclassical approximation. 
As a consequence, the results for the complete Hilbert space can be written as an integral collecting all the sectors, provided that the number of particles is large enough. We have shown than $N=50$ atoms are enough to guarantee the goodness of this approximation.

We have shown that each $j$-sector is equivalent to the one with $j=N / 2$, but with a smaller effective coupling strength. The main consequence is that, despite all of them have an ESQPT if the global coupling strength $\lambda$ is large enough, for any finite value $\lambda>\lambda_{c}$ there are a large number of $j$-sectors which are in the normal phase. To illustrate this fact, we have computed different magnitudes for different $j$ values: the density of states $\rho(E)$, the derivative of the density of states $\rho^{\prime}(E)$, the third component of the angular momentum $\left\langle J_{z}\right\rangle$, the derivative of the third component of the angular momentum $\left\langle d J_{z} / d E\right\rangle$, the temperature, $\beta$ and the first component of the angular momentum, $\left\langle J_{x}\right\rangle$.

We have analyzed the relationship between ESQPT and thermal phase transition when all the $j$-sectors are taken into account. This fact entails that the main signatures of the ESQPT, in particular the logarithmic singularities in the derivatives of the density of states and the expected value of $J_{z}$ are ruled out. However, $\left\langle J_{x}\right\rangle$ still changes from a value different from zero below the critical energy or the critical temperature, to zero above them. In particular, we have shown that the parity symmetry is spontaneously broken in the thermodynamical limit, if the system is in contact with a thermal bath (and thus, the canonical ensemble is used). Results obtained in this way coincide with the microcanonical calculations, if the integration in the phase space is restricted to one of the two disjoint regions existing when $E<E_{c}$. Parity symmetry becomes spontaneously broken at temperatures (or energies) at which the underlying semiclassical space is splitted in two regions; thermal fluctuations make the system choose one of the two existing disjoint regions.

Finally, we have also discussed the main physical differences between the Dicke model in isolation and in contact with a thermal bath. Despite both the microcanonical and canonical descriptions mainly coincide in the thermodynamic limit, one important difference remains. If the system is in contact with a thermal bath, that is, if it is described by means of the canonical ensemble, the energy $E_{*} / N=0$ constitutes an upper bound; this energy implies $T \rightarrow \infty$, and thus cannot be exceeded in any experiment. On the contrary, if the system remains thermally isolated and is heated by means of the Joule effect, for example by quenching $\lambda_{i} \rightarrow \lambda_{f} \rightarrow \lambda_{i}$ repeatedly, the limit $E_{*} / N=0$ can be exceeded; in other words, $E / N>0$ are accesible in the microcanonical description. 


\section{ACKNOWLEDGMENTS}

The authors gratefully acknowledge discussions with M. A. Bastarrachea-Magnani, S. Lerma-Hernández, J. G. Hirsch and P. Cejnar. A. R. is supported by Spanish Grants No. FIS2012-35316 and FIS2015-63770-P (MINECO/FEDER) and P. P. F. is supported by Spanish Grant No. FIS2014-53448-C2-1-P (MINECO/FEDER).

[1] S. Sachdev, Quantum Phase Transitions (Cambridge University Press, Cambridge, England, 1999).

[2] M. A. Caprio, P. Cejnar, and F. Iachello, Ann. Phys. (N.Y.) 323, 1106 (2008).

[3] P. Cejnar, M. Macek, S. Heinze, J. Jolie and J. Dobes, J. Phys. A 39, L515 (2006).

[4] P. Cejnar, and P. Stránský, Phys. Rev. E 78, 031130 (2008).

[5] P. Stránský, M. Macek, and P. Cejnar, Ann. Phys. 345, 73 (2014).

[6] P. Stránský, M. Macek, A. Leviatan, and P. Cejnar, Ann. Phys. 356, 57 (2015).

[7] W. D. Heiss and M. Müller, Phys. Rev. E 66, 016217 (2002); F. Leyvraz and W. D. Heiss, Phys. Rev. Lett. 95, 050402 (2005); P. Cejnar, M. Macek, S. Heinze, J. Jolie, and J. Dobes, J. Phys. A 239, L515 (2006).

[8] P. Ribeiro, J. Vidal, and R. Mosseri, Phys. Rev. E 78, 021106 (2008).

[9] A. Relaño, J. M. Arias, J. Dukelsky, J. E. García-Ramos, and P. Pérez-Fernández, Phys. Rev. A 78, 060102(R) (2008); P. Pérez-Fernández, A. Relaño, J. M. Arias, J. Dukelsky, and J. E. García-Ramos, Phys. Rev. A 80, 032111 (2009); L. F. Santos, M. Távora, and F. Pérez-Bernal Phys. Rev. A 94, 012113 (2016).

[10] P. Pérez-Fernández, A. Relaño, J. M. Arias, P. Cejnar, J. Dukelsky, and J. E. García-Ramos, Phys. Rev. E 83, 046208 (2011); P. Pérez-Fernández, A. Relaño, J. M. Arias, P. Cejnar, J. Dukelsky, and J. E. García-Ramos, Phys. Rev. E 83, 046208 (2011).

[11] T. Brandes, Phys. Rev. E 88, 032133 (2013).

[12] J. M. Arias, J. Dukelsky, and J. E. García-Ramos, Phys. Rev. Lett. 91, 162502 (2003); P. Cejnar, J. Jolie, and R. F. Casten, Rev. Mod. Phys. 82, 2155 (2010).

[13] F. Pérez-Bernal and F. Iachello, Phys. Rev. A 77, 032115 (2008); F. Pérez-Bernal and O. Álvarez-Bajo, Phys. Rev. A 81, 050101(R) (2010). 
[14] A. Relaño, J. Dukelsky, P. Pérez-Fernández, and J. M. Arias, Phys. Rev. E 90, 042139 (2014).

[15] V. M. Bastidas, P. Pérez-Fernández, M. Vogl, and T. Brandes, Phys. Rev. Lett. 112, 140408 (2014).

[16] R. Puebla, M.-J. Hwang, and M. B. Plenio, Phys. Rev. A 94, 023835 (2016).

[17] D. Larese and F. Iachello, J. Mol. Struct. 1006, 611 (2011); D. Larese, F. Pŕez-Bernal, and F. Iachello, J. Mol. Struct. 1051, 310 (2013); D. Larese, M. A. Caprio, F. Pérez-Bernal, and F. Iachello, J. Chem. Phys. 140, 014304 (2014); N. F. Zobov, S. V. Shirin, O. L. Polyansky, J. Tennyson, P.-F. Coheur, P. F. Bernath, M. Carleer, and R. Colin, Chem. Phys. Lett. 414, $193(2006)$.

[18] B. Dietz, F. Iachello, M. Miski-Oglu, N. Pietralla, A. Richter, L. von Smekal, and J. Wambach, Phys. Rev. B 88, 104101 (2013).

[19] L. Zhao, J. Jiang, T. Tang, M. Webb, and Y. Liu, Phys. Rev. A 89, 023608 (2014).

[20] R. Puebla, A. Relaño, and J. Retamosa, Phys. Rev. A 87, 023819 (2013); R. Puebla and A. Relaño, Europhys. Lett. 104, 50007 (2013).

[21] R. Puebla and A. Relaño, Phys. Rev. E 92, 012101 (2015).

[22] P. Cejnar and P. Stránský, Phys. Scr. 91, 083006 (2016).

[23] K. Huang, Statistical Mechanics, Wiley (1987).

[24] P. Stránský and P. Cejnar, Phys. Lett. A 381, 984 (2017).

[25] M. A. Bastarrachea-Magnani, S. Lerma-Hernández, J. G. Hirsch, J. Stat. Mech 093105 (2016).

[26] R. H. Dicke, Phys. Rev. 9399.

[27] H. J. Carmichael, C. W. Gardiner and D. F. Walls, Phys. Lett. 46 A 47 (1973).

[28] G. Comer Duncan, Phys. Rev. A 9, 418 (1974).

[29] C. Emery and T. Brandes, Phys. Rev. E 67066203 (2003); C. Emery and T. Brandes, Phys. Rev. Lett. 90044101 (2003).

[30] M. J. Hartmann, F. G. S. L. Brandäo, and M. B. Plenio, Nat. Phys 2, 849 (2006); A. D. Greentree, C. Tahan, J. H. Cole, and L. C. L. Hollenberg, Nat. Phys. 2, 856 (2006).

[31] K. Baumann, C. Guerlin, F. Brennecke, and T. Esslinger, Nature (London) 464, 1301 (2010).

[32] M. Aparicio Alcalde, M. Bucher, C. Emary, and T. Brandes, Phys. Rev. E 86, 012101 (2012).

[33] A. Altland and F. Haake, New. J. Phys. 14, 073011 (2012).

[34] M. A. Bastarrachea-Magnani, S. Lerma-Hernández, and J. G. Hirsch, Phys. Rev. A 89, 032102 (2014). 
[35] P. Stránský and P. Cejnar, Phys. Lett. A 380, 2367 (2016).

[36] A. Relaño, M. A. Bastarrachea-Magnani, and S. Lerma-Hernández, EPL 11650005 (2016); M. A. Bastarrachea-Magnani, A. Relaño, S Lerma-Hernández, B López-del-Carpio, J ChávezCarlos, and J G Hirsch, J. Phys. A 50, 144002 (2017). 